\title{
Performance Evaluation of Location Management Techniques in PCS Network
}

\author{
Sarvpal H. Singh \\ Associate Professor \\ MMM Engg. College, Gorakhpur
}

\author{
Sudhir Agrawal \\ Head (CSE) \\ BIT, GIDA, Gorakhpur
}

\author{
Vijay Kumar \\ Associate Professor \\ DDU Gorakhpur University, Gorakhpur
}

\begin{abstract}
Location management being a major part of cellular network provides mobility to mobile subscribers. The current location information is required to route the call correctly to the recipient irrespective of its current location. The current HLR/VLR architecture for location management in standards like IS-41 \& GSM suffers from call setup delay due to network congestion and also prone to failure due to its centralized nature. This paper attempts to make an evaluation of the optimized location management techniques suggesting fully distributed, hierarchical and multi HLR approach against the conventional one and also highlights the pros and cons of all the reviewed techniques.
\end{abstract}

\section{Keywords}

Mobility Management, Location Management (LM), Location Area (LA)

\section{INTRODUCTION}

The cellular system is getting emerged as a fastest growing field and has become a hot area for researchers' community. The standard like GSM has dominated the world communication market through its widespread use in European countries, India etc. In GSM, a Mobile Unit (MU) is allowed to move freely within the entire area of coverage. Due the random movement of mobile unit, its geographical location is unpredictable. To deliver a call correctly to MU, it is necessary to have information about its current location. This requirement has been implemented in GSM standard through HLR/VLR architecture [1-3]. The entire process of the mobility management component of the cellular system is responsible for two tasks:(a) Location management- that is, identification of the current geographical location or current point of attachment of a mobile unit which is required by the MSC (Mobile Switching Center) to route the call- and (b) Handoff- that is, transferring (handing off) the current (active) communication session to the next base station, which seamlessly resumes the current session using its own set of channels. Thus, this whole location management process is a kind of directory management problem where current locations of MU are maintained continuously by carrying out updations in HLR with the help VLR. The current point of attachment or location of a subscriber (mobile unit) is expressed in terms of the cell or the base station to which it is presently connected. The mobile units (called and calling subscribers) can continue to talk and move around in their respective cells; but as soon as both or any one of the units moves to a different cell, the location management procedure is invoked to identify the new location.

The provision of unrestricted mobility of mobile units creates a complex dynamic environment, and the location management component must be able to identify the correct location of a unit without any noticeable delay. The location management performs three fundamental tasks: (a) location update, (b) location lookup, and (c) paging. In location update, which is initiated by the mobile unit, the current location of the unit is recorded in HLR and VLR databases. Location lookup is basically a database search to obtain the current location of the mobile unit and through paging the system informs the caller the location of the called unit in terms of its current base station.

The MSC initiates these two tasks. Understanding the importance and role of location management, it is always desired to have efficient location management so as to provide communication facilities to clients during mobility. Efforts are continued to explore new strategies to reduce the handshaking overheads, improve the bandwidth usage and facilitating the call to subscriber with better and better response time.

The continuous location trapping of MU has made it desirable to have the efficient location management schemes so that HLR updations' overhead can be minimized. The other related issue is the distribution of HLR to shorten the access path, which is similar to data distribution problem in distributed database systems. Moreover, HLR may proved to be a single point failure for the entire network. These issues have motivated research community greatly to develop and explore more and more innovative techniques for location management of mobile units. Recently a number of innovative location management schemes have appeared in the research world.

The approaches developed in the area of cellular location management can broadly be classified as (1) Centralized approaches (2) Distributed approaches. Centralized approach [1-3] keeps information only on one node in the mobile network. For example, existing location management standards, IS-41 and GSM are centralized approaches. Location lookup and update operations are simple in this case but they suffer from severe problems like congestion, central point failure etc. In Distributed approaches [8-10] user information is distributed among many nodes in the network. This has better stability in comparison to centralized approach but location lookup and update operations are somewhat complex in this case. However, many schemes have come forward to solve these problems. A variant of distributed approach is hierarchical approach [10-15] which has been suggested to overcome the drawbacks of centralized approach. In this approach, location registers in the PCS network are arranged in hierarchical fashion. The topology resembles to a tree with a root level Location Register (LR) and its ascendant LRs while each leaf level LR performs location management operation for one zone of PCS network.

Looking at the need of efficient location management, this paper attempts to make a comparative analysis of the modified HLR/VLR centralized approach referred to as Multi HLR approach, distributed approach and conventional centralized HLR/VLR approach. The remaining part of this paper has been organized as follows: Section 2 gives an overview about centralized HLR/VLR approach used in IS-41 and GSM. Section 3 sheds light on distributed approach. The Multi HLR approach is discussed in Section 4. A comparative performance analysis has been carried out 
in Section 5 which is followed by the final results in section 6 and conclusion in section 7 .

\section{CENTRALIZED APPROACH}

This section describes existing standard of location management followed by the approaches evolved to overcome drawbacks of existing standard.

The two popular standards currently used are GSM and IS-41. They make use of two types of registers, home location register (HLR) and visitor location register (VLR), to store the location information of the mobile terminals. Figure 1 shows the basic architecture [15] under this two-level hierarchy.

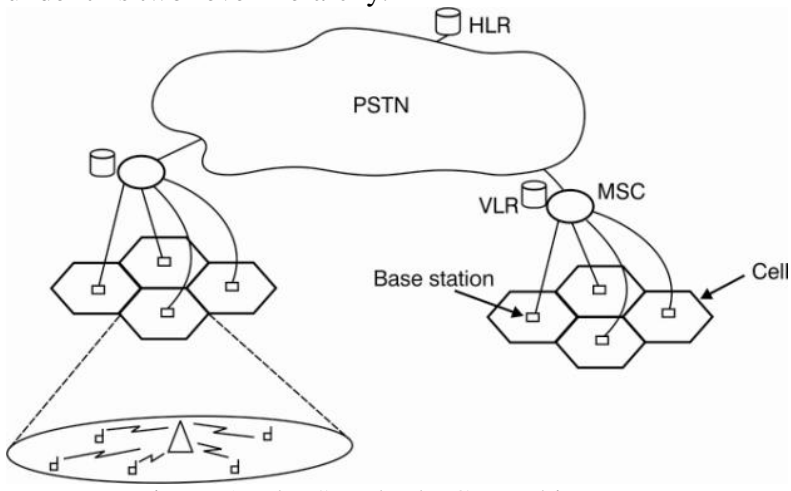

Figure 1: The Standard PCN architecture

The HLR stores the user profiles of its assigned subscribers. These user profiles contain information such as the type of services subscribed, the quality-of-service (QoS) requirements and the current location of the mobile terminals. Each VLR: stores replications of the user profiles of the subscribers currently residing in its associated RA. In order to effectively locate a mobile terminal when a call arrives, each mobile terminal is required to report its location whenever it enters a new RA. This reporting process is called location update. On receiving a location update message, the MSC updates its associated VLR and transmits the new location information to the HLR. We call this Register update process as location registration. The HLR will acknowledge the MSC for the successful registration and it will also deregister the mobile terminal at the VLR of old RA. In order to locate a mobile terminal for call delivery, the HLR is queried to determine the serving MSC of the target mobile terminal. The HLR then sends a message to this MSC which, in turn, will determine the serving base station of the mobile terminal by paging all cells within its associated RA.

This location tracking scheme requires the exchange of signaling messages between the HLR and the new and old MSC's whenever the mobile terminal crosses an RA boundary. This may result in significant traffic load to the network especially when the current location of the mobile terminal is far away from its HLR and the mobile terminal is making frequent movements among RA's. Besides, the HLR may experience excessively high database access traffic as it is involved in every location registration and call delivery. This may result in increased connection set up delay during periods of high network utilization.

Associated problems: Since every location request as well as location registration are serviced through an HLR, it becomes overloaded. Due to this reason, traffic on the links leading to the HLR is heavy, which increases time required to establish connection to the MU. HLR is a single point of failure in the network as any HLR system failure causes all mobiles registered with HLR to be unreachable even though mobile host may be roaming and away from HLR region. The centralized approaches do suffer with an additional problem. Though the callee (mobile unit who is called) is located in the region near to the caller, its location information is needed to be obtained from HLR which can be placed over a long geographical distance. This is referred to as Tromboning problem. Various strategies have been suggested by researchers to optimize the centralized approach. The Local Anchor (LA) Scheme, proposed by $\mathrm{Ho}$ and Akyildiz [5], reduces the signaling traffic by using a local anchor (a VLR, a mobile user is currently visiting when he/she receives a call). In this scheme, a VLR close to the user is selected as the local anchor (LA) for the user. Whenever a user moves from one RA to another, it will perform location update to the LA. A LA for a mobile will change only when a call request to the mobile arrives; at the same time, the HLR is also updated via the registration process. When a call request terminating at this user is received by the HLR, the user can be traced to the LA. The LA scheme avoids update to HLR completely at the expense of the increase in local signaling traffic. The drawback of this scheme is that when the user keeps moving constantly without receiving any call, the updates to LA may become costly, a similar bottleneck as the HLR. Another scheme, Per-user Pointer Forwarding Scheme [6] proposed by Jain and Lin., in which, some updates to the HLR can be avoided by setting up a forwarding pointer from the previous VLR to the new VLR. When a call request to a mobile user arrives, the PCS network first queries the user's HLR to determine the VLR, which the user was visiting at the previous location update, then follows a chain of forwarding pointers to the user's current VLR to find the mobile user. The traffic to the HLR is decreased by using the pointer chain; however, the penalty is the time delay for tracking the user when a call to the user arrives. The longer the pointer chain, the less the signaling traffic, the longer the setup delay for finding the user. To avoid long setup delay, a threshold of the length of the pointer chain is used. The user needs to perform registration to the HLR after the chain threshold is reached. In order to overcome the drawbacks these two schemes, two-level pointer forwarding strategy was proposed by Ma and Fang [7]. Two kinds of pointers are used in this scheme. Some VLRs are selected as the Mobility Agents (MA), which will be responsible for location management in a larger area comparing to the RAs and can be geographically distributed.

Instead of always updating to the HLR, which would become the bottleneck otherwise, many location updates are carried out in the mobility agents. Thus, the two-level pointer forwarding scheme is designed to reduce the signaling traffic: pointers can be set up between VLRs as the traditional pointer forwarding scheme and can also be set up between MAs. The results has shown that this strategy can significantly reduce the network signaling traffic for users with low Call to Mobility Ratio (CMR)[6-7] without increasing much of the call setup delay. Ratnam, Matta and Rangrajan have suggested caching [11] for current standards. In existing HLR/VLR scheme, call is routed through MSC to LR in which callee is located. When a particular MSC receives a large number of calls to a particular mobile that belongs to a different home system, the signaling and database cost involved in setting up the call can be significantly reduced by caching the location information at the calling MSC. Each time when a call is attempted, the cached information is checked first. Since the access time in looking up an entry in the cached memory is very short (order of microseconds), checking the cached information for every call doesn't affect the performance of 
the MSC. In case of cache hit, call is directly routed to serving LR of callee. But in case of cache miss, HLR is needed to be contacted and call-establishing time will be longer than normal HLR/VLR scheme.

\section{DISTRIBUTED APPROACHES}

The approaches of this category can be further classified in the categories of fully and partially (hierarchically) distributed. A novel approach for efficient location management is proposed by Ratnam, Matta and Rangrajan [9] in which the idea is to fully distribute the location information across Location Registers (LRs). These LRs replace the centralized VLRs and HLRs which are found in current PCS networks.

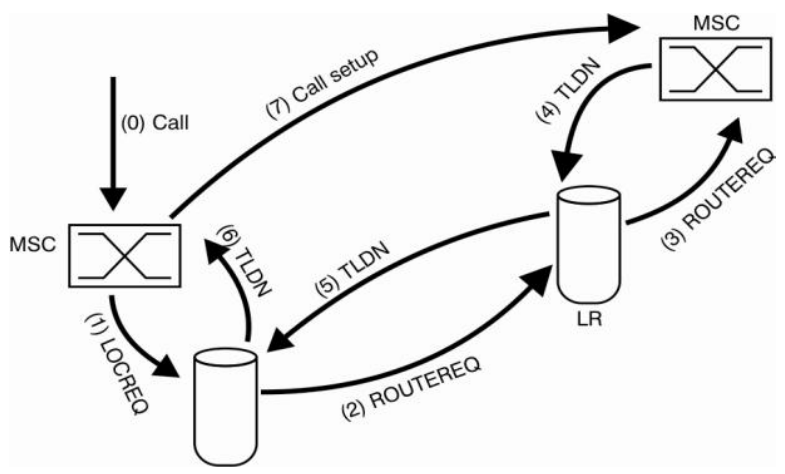

Figure 2: Call Delivery in Fully Distributed Location Management

Thus, this scheme proposes a unique feature of availability (fault-tolerance) by not having the concept of "home." Since there are no HLRs or VLRs in this system, each LR maintains the location information of not only the mobiles that are local to it, but also of other mobiles in the network, i.e., the location information of all mobile hosts are fully replicated in all the LRs. The LRs are distributed throughout the network. An LR may serve one or more MSCs just like the VLR in the PCS architecture. LRs function as both the location registry for the local mobile hosts as well as the lookup directory for the location of other mobile hosts. The type of location information maintained for a mobile host depends on whether the mobile is local to the LR or not. For local mobile hosts, LR maintains the id of the MSC that is currently serving the mobile. For mobile hosts that are not local, LR maintains the id of the LR where the mobile host currently resides. When a mobile registers with an LR, the new location information is disseminated to all other LRs in the network. This dissemination is carried out in parallel through the whole network so that the new location is very quickly updated at all LRs. When a call request arrives at the local LR, this LR can directly contact the serving LR (Figure 2), thus avoiding the tromboning problem present in the current IS-41 standard. This scheme requires that new location information about all mobiles be disseminated to all the LRs in the network. As the size of the network grows, location information dissemination not only consumes a significant portion of the network bandwidth but also consumes significant portion of LR resources to process large number of update messages. In addition, the gain of employing full dissemination diminishes with the size of the network. Full location information dissemination can be avoided by logically arranging LRs in a hierarchical fashion i.e. a tree structure. The idea is to divide the LRs into hierarchy of clusters, and confine location information dissemination to within the clusters as much as possible. In this scheme [9] the goal is not only to reduce the overhead of location management, but also to uniquely provide high availability through (selective) replication of location information.

Figure 3 shows the conceptual arrangement of the LRs in a hierarchical network. This approach uses a distributed location management. Each LR maintains the location information of all the mobiles that are currently being served in the sub tree rooted from the LR. It also maintains the location of the mobiles that belong to the sub tree rooted from its sibling LRs. Note that the sub tree rooted from a leaf node contains only that leaf node. If a mobile host is being served by one of the descendants of an LR, then the LR maintains the ID of its immediate child LR, whose sub tree contains the mobile host, to track the mobile host. Tracking the LR serving a mobile host involves traversing the LR tree hop-by-hop until the serving LR is reached. If the location entry for a mobile host does not exist in an LR, then the tracking request is forwarded to the LR's parent LR. In this way the tracking request traverses the tree upwards until the LR which has the location information for the mobile host is reached. That LR forwards the tracking request to the LR pointed to by the location information. Here, location tracking traverses laterally. From there, it traverses downwards until the LR currently serving the mobile host is reached.

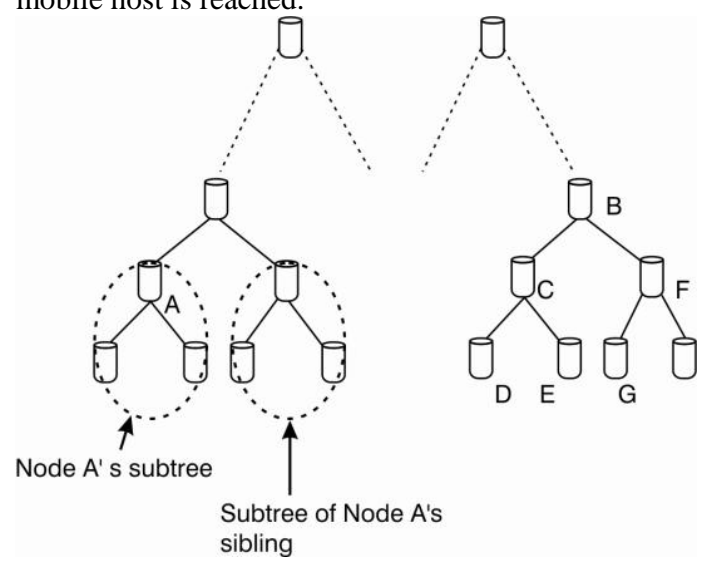

Figure 3: Conceptual diagram showing the hierarchical arrangement

Figure 4 shows hierarchical location management structure discussed by Suh, Choi and Kim [10] having R layers. The hierarchically distributed servers have database for keeping information of mobile's location. The mobile enrolls in one of the layer1 servers called mobile's home server. To the ancestor servers of the mobile's home server, the mobile is regarded as home mobile. A server regards a mobile as foreign mobile which is located under server's coverage but whose home server is outside this server. The mobile identification (ID) is composed of hierarchical area address and terminal number. Server on layer 1 has location information about address of the parent server on layer 2 and address of mobile's residing LA and server on layer $\mathrm{i}(\mathrm{i}=2,3 \ldots \mathrm{R})$ has location information about address of parent server on layer $i+1$, address of the server on layer i-1 under which the mobile is and address of the server on layer i-1 under which the mobile is. Consequently, information stored at the servers represents 'tracking path' for each mobile from its home server to its currently residing LA. The terminal movement from the old LA to the new LA triggers the location updates at the servers on hierarchical structure.

For call delivery, there are different strategies depending on how to forward the call to the callee on hierarchical nodes [10]. The first scheme referred to as Home server first scheme (HSF), the call is passed directly to 
callee's home server. This scheme is advantageous when the mobiles have a tendency of staying around their home server areas.

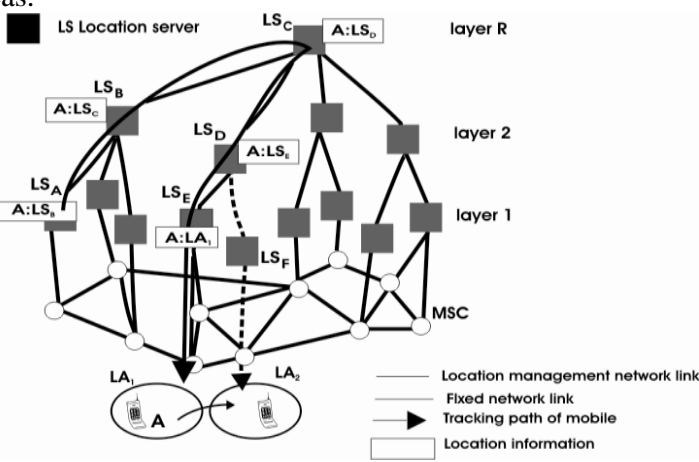

Figure 4 : Hierarchical location management system structure

Since mobile ID consists of hierarchical area address, the address of callee's home server can be easily found at caller's switch. In second scheme called Least common ancestor (LCA) server first scheme when a mobileterminated call occurs, caller's switch does not figure out the switch of callee's current location. Thus caller's switch forwards the call to the appropriate ancestor server depending on how far it is away from callee's home. According to the LSF scheme, the call is passed directly to the LCA server of the caller's switch and callee's home server. The address of LCA server can be easily found by comparing the area addresses of caller's layer 1 location server and callee's home server. The third scheme called Distributed LSF scheme is based on 2 layered location management structure at which location servers are located. Layer 2 servers are logically connected together with each other as shown in Figure 5. Mobile layer 2 ancestor server is called layer 2 home server and contain information like address of layer 2 server under which mobile is located, address of layer server under which the mobile is located at this moment and address of layer 1 server under which the mobile is located at this moment. The layer 1 server has same information as that of previous schemes. Thus when a mobile moves between LAs of the different layer 1 location servers, the location update occurs at the new layer 1 location server and the layer 2 location server(s).

According to distributed LSF strategy, when a call occurs, the call is forwarded to the closest home server of callee. In other words, when a call is initiated under callee's layer 1 home server, it is forwarded to callee's layer 1 home server. When a call is initiated under the area but callee's layer 1 home server's coverage, it is forwarded to layer 2 home server of the callee. Then, the call is forwarded to callee's current location server through the tracking path.

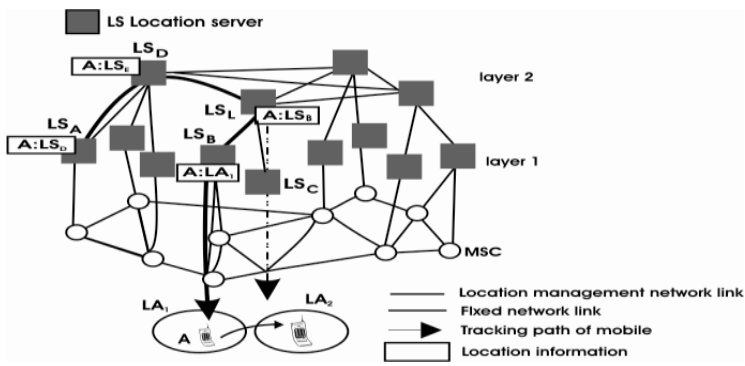

Figure 5: Call Delivery procedure of LSF scheme

\section{MULTI HLR APPROACH}

For improving location management in PCS network a new concept suggested by Haider Safa and Pierre employs Multi HLR [12] as opposed to ONE HLR in IS-41 and GSM models. Indeed this new architecture associates with each MU (mobile unit) two types of HLR; a resident HLR that serves the location areas in which the MU often resides and a serving HLR that serves the MU when it is roaming outside its resident HLR covering area.

\section{Description of multi HLR architecture}

The Multi HLR architecture employs two HLRs instead of one HLR as shown in Fig. 6. Each HLR may serve several VLRs. A VLR can serve one or more LAs but it can be served only by one HLR. For each MU two types of HLRs are defined: a resident HLR and a serving HLR. The MU's resident HLR covers the LAs in which the MU often resides. It stores permanently the MU location information and parameters. The MU's serving HLR serves the MU when it is roaming outside its resident HLR covering area. When the MU moves to a new LA not served by its resident HLR, the HLR that serves this LA becomes the MU's serving HLR.

When an MU is called, the VLR of the calling unit verifies if the called MU is local. If it is, then the called MU is located. Otherwise, the VLR forwards the call request to its HLR which verifies whether the called MU's is roaming in its covering area but under different VLR or in the covering area of another HLR. Then this HLR forwards the call request to the appropriate network entity.

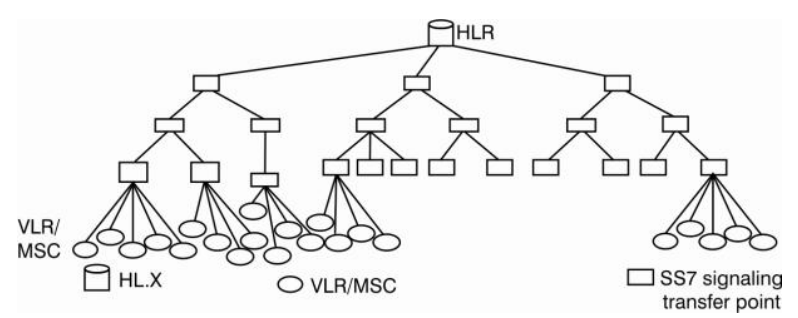

Figure 6: Conventional HLR/VLR architecture

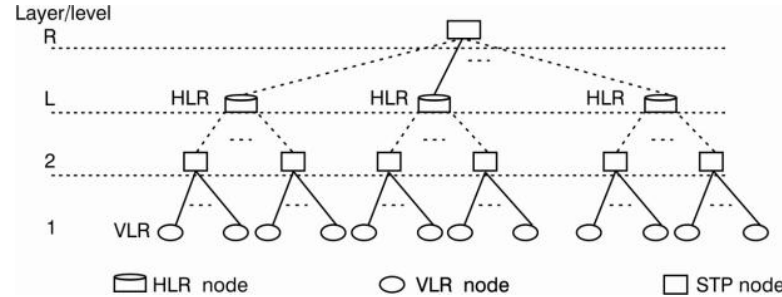

Figure 7: Multi HLR architecture

Location update procedure: This architecture distinguishes between several types of MU moves: intra-VLR move, intraHLR move and inter-HLR move. The location update scenarios associated with these moves are as follows Intra-VLR move: This move occurs when the MU moves between two LAs that belong to the same VLR. The MU's location profile is then updated only at the VLR level.

Intra-HLR move: This move occurs when the MU moves between two LAs served by two different VLRs but within the covering area of the same HLR. Its main steps, shown in Figure 8, are described as following:

- The MU moves to a new LA served by a different VLR and registers with this VLR.

- The VLR of the new LA sends a location update request to its HLR. 
- The HLR, in its turn, sends a location cancellation request to the VLR of the old LA.

- The old VLR sends a location cancellation acknowledgment to the HLR.

- Upon receiving this acknowledgment, the HLR acknowledges the location update to the new VLR, which instructs the new LA to start serving the MU.

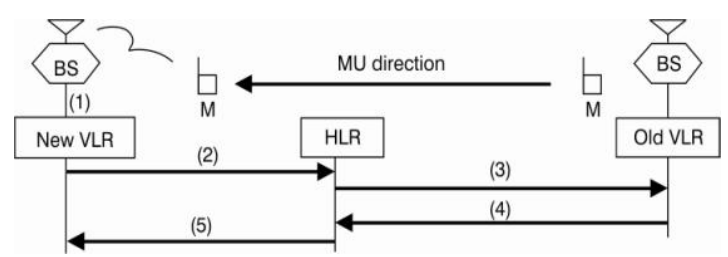

Figure 8: Location update procedure of an intra-HLR move

Inter-HLR move: This move occurs when the MU between two Las are served by two VLRs that belong to two different HLRs. In this context, three cases are studied:

(a) The MU leaves the covering area of its resident HLR and enters the covering area of another HLR. This new HLR becomes the MU's serving HLR.

(b) The MU returns to its resident HLR, i.e. the MU returns to the covering area of its resident HLR from the covering area of another HLR.

(c) The MU moves between two distinct HLRs that are different than its resident HLR.

The main steps of this scenario are described as following:

Step 1: The MU enters a new LA and registers with the VLR of this LA.

Step 2: If the case (a) prevails, then:

- The VLR of the new LA sends a location update request to its HLR. This HLR becomes the serving HLR for the MU (serving HLR 1 in Figure 9).

- $\quad$ The serving HLR sends a location update request to the MU's resident HLR.

- The resident HLR sends a registration cancellation request to the old VLR.

- The old VLR sends a cancellation acknowledgment to the resident HLR.

- Upon receiving this acknowledgment, the resident HLR updates the profile of the MU and sends a location update acknowledgment to the serving HLR. The serving HLR, in its turn, sends a registration acknowledgment to the current VLR of the MU. Then this VLR starts to serve the MU.

Step 3: If the case (b) prevails, then:

- The MU's new VLR sends a location update request to its HLR, which is the MU's resident HLR.

- The resident HLR forwards this request to the MU's old serving HLR.

- The old serving HLR sends a cancellation request to the MU's old VLR.

- The old VLR acknowledges the cancellation request.

- The old serving HLR forwards the acknowledgment to the MU's resident HLR then it deletes the MU profile. The resident HLR updates the MU profile.

The resident HLR, in its turn, sends a location update acknowledgment to the VLR of the new LA, which starts, in its turn, serving the MU.

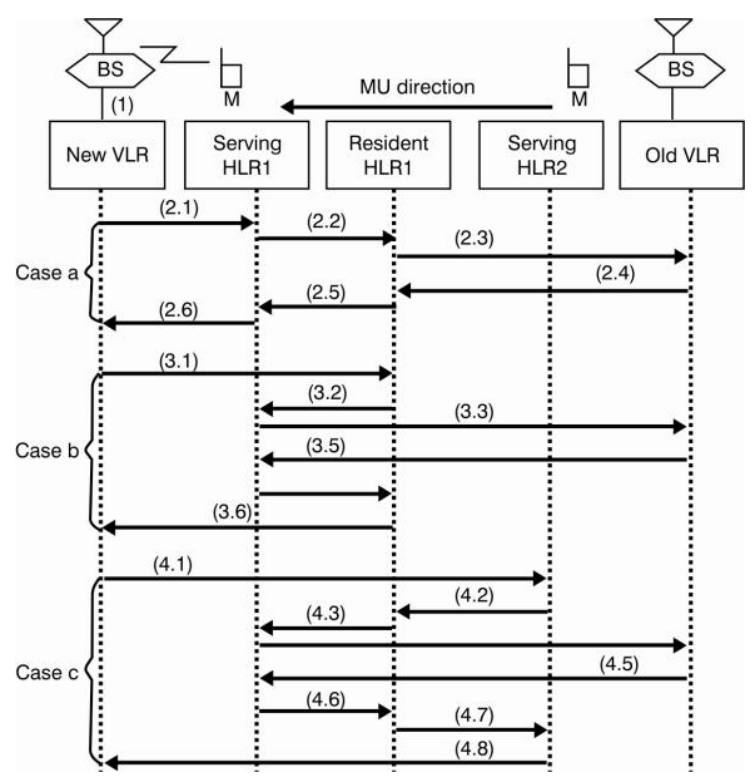

Figure 9: Location update procedure for an inter-HLR move

Step 4: If the case (c) prevails, then:

- The VLR of the new LA sends a location update request to its HLR. This HLR becomes the MU's new serving HLR (Serving HLR2 in Figure 9).

- The new serving HLR sends a location update request to resident HLR. The resident HLR updates the MU profile to indicate its new serving HLR.

- The MU's resident HLR, in its turn, sends a registration cancellation request to the MU's old serving HLR (Serving HLR1 in Figure 9).

- The old serving HLR forwards the cancellation request to the MU's old VLR.

- The old VLR sends a cancellation acknowledgment to the old serving HLR.

- Upon receiving this acknowledgement, the old serving HLR forwards the acknowledgement to the MU's resident HLR and deletes the MU profiles.

- The resident HLR acknowledges the location update to the new serving HLR which updates the MU profile to indicate the VLR of its new LA.

- The new serving HLR sends a location update acknowledgment to the new VLR. Upon receiving this acknowledgment, the new VLR starts serving the MU.

\section{Location search procedure:}

An algorithm that illustrates the location search procedure in this model is presented in Figure 10. The main purpose of this procedure is to determine the MU's current LA in order to deliver a call. For sake of simplicity, we assume that the MU, N is calling the MU, $\mathrm{M}$. The algorithm considers the following three possible scenarios:

1. $\quad \mathrm{M}$ and $\mathrm{N}$ are served by the same VLR: The call is local and is handled by the VLR.

2. $\quad \mathrm{M}$ and $\mathrm{N}$ are served by two different VLRs but both VLRs are served by the same HLR.

3. $\mathrm{M}$ and $\mathrm{N}$ are served by two different VLRs that belong to two different HLRs: In this context, the following cases are possible:

- $\quad \mathrm{M}$ is roaming in the covering area of its resident HLR.

- $\quad \mathrm{N}$ is roaming in the covering area of M's resident HLR but $M$ is not in its resident HLR. 
- $\quad \mathrm{M}$ and $\mathrm{N}$ are roaming outside of M's resident HLR.

If ( $\mathrm{N}$ and $\mathrm{M}$ are served by the same VLR) then Local Call

Else \{

N's VLR sends a call request to its HLR,

If (N's VLR and M's VLR are served by the same HLR) then \{

HLR transfers the call request to M's VLR.

M's VLR instructs the MSC to assign a TLDN for the call and returns it to the HLR in an acknowledgment message.

The HLR forwards this TLDN to N's VLR.

\}

Else if ( $\mathrm{M}$ is roaming in the covering area of its resident HLR) then \{

N's serving HLR forwards the query to M's resident HLR.

M's resident HLR forwards the request to M's current VLR.

M's VLR returns a TLDN for the call to M's resident HLR.

M's resident HLR forwards it to N's serving HLR.

N's serving HLR transfers it to the N's current VLR. \}

Else if (N's serving HLR is M's resident HLR) then \{

M's resident HLR, which is N's HLR, determines M's serving HLR and forwards the call to it.

M's serving HLR transfers the call to the M's current VLR.

M's VLR assigns a TLDN for the call and returns it in an ack message to M's serving HLR.

M's serving HLR forwards it to the calling HLR (i.e., N's serving HLR)

N's serving HLR forwards this TLDN to N's current VLR.

\}

Else \{

N's serving HLR transfers the query to M's resident HLR.

M's resident HLR determines M's serving HLR then forwards the call to it.

M's serving HLR transfers the request to M's current VLR.

M's VLR assigns a TLDN to the call and returns it in an ack msg. to the M's serving HLR.

M's serving HLR forwards it to M's resident HLR.

M's resident HLR forwards this TLDN to N's serving HLR.

N's HLR transfers the response to N's current VLR. \}

Then the communication is established between the N's LA and M's LA.

Figure 10: Location search algorithm

(M: callee MU, N: calling MU)

\section{PERFORMANCE ANALYSIS}

An analytical model to evaluate the performance of the Multi HLR, conventional HLR/VLR, the database driven and hierarchical architectures are done in this section. In this analysis, a hierarchical tree of R layers is used, as shown in Figure 7. The layer $\mathrm{R}$ contains the root node and the layer 1 contains the leaf nodes. In the database driven scheme proposed in [13-15], a database is installed on each node of the tree and the MUs are assigned to the leaf nodes. In the HLR/VLRs scheme, the network database, HLR, is situated on the only node of layer R and the VLRs are installed on the leaf nodes. Here, the HLRs are installed on the nodes of layer $\mathrm{L}(1<\mathrm{L}<\mathrm{R})$, while the VLRs remains installed on the leaf nodes. The following terms are used in performance analysis :-

$\mathbf{m}_{\mathbf{x}, \mathbf{y}}$ - Layer of the closest common node to LA $\mathrm{x}$ and LA $\mathrm{y}$.

p - Probability that the MU move is intra-VLR.

q - Probability that the called and the calling MUs are served by the same VLR.

$\alpha$ - Probability that the MU move is inter-HLR.

$\boldsymbol{\beta}$ - Probability that the MU's resident HLR is involved in the inter-HLR move, i.e., the MU leaves or returns to its resident HLR covering area.

$\delta$ - Probability that the calling MU and the called MU are roaming in the covering areas of two different HLRs.

n - New LA of the MU.

a - Old LA of the MU.

S - LA of the calling unit (source).

D - LA of the called MU (destination).

$\boldsymbol{\theta}$ - Probability that the call is originated from or terminated at the called MU's resident HLR covering area.

$\mathrm{P}\left(\mathbf{m}_{\mathbf{x}, \mathbf{y}}=\mathrm{i}\right)$ is defined as the probability that the closest common node to LA $\mathrm{x}$ and LA $\mathrm{y}$ is in layer $\mathrm{i}$. This probability can be given by the following equation.

$$
\begin{aligned}
\mathrm{P}\left(\mathrm{m}_{\mathrm{a}, \mathrm{n}}=\mathrm{i}\right)= & \underset{(1-\mathrm{p})^{\mathrm{i}-1} \text { for } \mathrm{i}=\mathrm{R}}{\mathrm{i}-1} \text { for } \mathrm{i}=1,2 \ldots \ldots \ldots . . \mathrm{R}-1 \\
\mathrm{P}\left(\mathrm{m}_{\mathrm{s}, \mathrm{d}}=\mathrm{i}\right)= & \mathrm{q}(1-\mathrm{q})^{\mathrm{i}-1} \text { for } \mathrm{i}=1,2 \ldots \ldots \ldots \ldots \mathrm{R}-1 \\
& (1-\mathrm{q}) \mathrm{i}-1 \text { for } \mathrm{i}=\mathrm{R}
\end{aligned}
$$

The costs of various operations used in this analysis are denoted as follows:-

$\mathbf{T}(\mathrm{i}, \mathrm{j})$ - Cost of transmitting a message over a link between two adjacent layers $i$ and $j$.

$\mathbf{C}_{\mathbf{m}}(\mathrm{i})$ - Cost of accessing or updating a database in layer i.

$\mathbf{M}_{\text {multi HLR }}$ - Estimated cost of a location update in the mult HLR scheme.

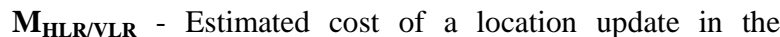
HLR/VLRs architecture

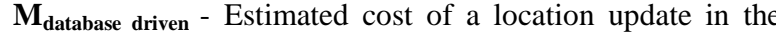
database driven architecture.

$\mathbf{M}_{\text {hierarchical }}$ - Estimated cost of a location update in the hierarchical architecture.

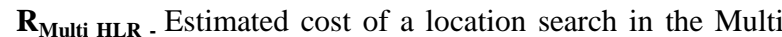
HLR scheme.

$\mathbf{R}_{\text {HLR/VLRs }}$ - Estimated cost of a location search in the HLR/VLRs architecture

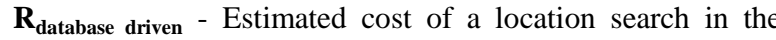
database driven architecture.

$\mathbf{R}_{\text {hierarchical }}$ - Estimated cost of a location search in the hierarchical architecture.

The estimated cost of a location update in the Multi HLR scheme is given by:

$$
\begin{aligned}
M_{\text {MultitLLR }}= & P\left(m_{a, n}=1\right) \times C_{m}(1)+\sum_{i=2}^{L} P\left(m_{a, n}=i\right) \times\left\{2 C_{m}(1)+C_{m}(L)+4 T(1, L)\right\} \\
+ & \propto\left\{\left\{2 C_{m}(1)+C_{m}(L)+4 T(1, L)+\sum_{i=L+1}^{R} P\left(m_{a, n}=i\right)\right.\right. \\
& \left.\times\left[\beta \times\left(\sum_{j=L}^{i-1} 4 T(j, j+1)+C_{m}(L)\right)+(1-\beta) \times\left(\sum_{j=L}^{i-1} 8 T(j, j+1)+2 C_{m}(L)\right)\right]\right\} \\
\text { where: } & \quad \times 1-\sum_{i=1}^{L} P\left(m_{a, n}=i\right)
\end{aligned}
$$

In Eq. 3, the first part illustrates the cost of the location update procedure of an intra-VLR move and intra-HLR move. The second part illustrates the scenario after an inter- 
HLR move. $\mathrm{T}(1, \mathrm{~L})=\mathrm{T}(1,2)+\mathrm{T}(2,3)+\ldots+\mathrm{T}(\mathrm{L}-1, \mathrm{~L})$ is equal to the cost of traversing links between a node of layer 1 (i.e., VLR) and the node of layer L (i.e., where an HLR is located in the multi HLR scheme). This cost is multiplied by 4 because, when a signaling message is sent from a VLR to the HLR, the latter sends a similar message to the old VLR. By adding the cost of the acknowledgment from the old VLR to the HLR and then from the HLR to the current VLR, we can justify the $4 \mathrm{~T}(1, \mathrm{~L})$. Similar analysis applies on transmitting costs in second part of the equation.

For comparison purposes, the costs of the HLR/VLRs, database driven architecture and hierarchical architecture are needed. In IS-41 based scheme, movement between LA's under the same VLR makes location updates at VLR only. For inter-VLR movement, HLR (i.e. layer R server) and both old and new VLRs update location information. Hence, the estimated cost of the location update of the HLR/VLRs architecture is as follows:

$$
\begin{aligned}
M_{H L R / V L R}= & P\left(m_{a, n}=1\right) \times C_{m}(1)+ \\
& \sum_{i=2}^{R} P\left(m_{a, n}=i\right) \times\left\{2 C_{m}(1)+C_{m}(R)+4 T(1, R)\right\}
\end{aligned}
$$

In the database driven architecture, the location update occurs at all the databases on the path between the old LA and the new LA. Therefore, the estimated cost of a location update operation in this scheme can be written as follows:

$$
M_{\text {Databasedriven }}=\sum_{i=1}^{R} P\left(m_{a, n}=i\right) \times\left\{\sum_{j=1}^{i-1}\left[4 T(j, j+1)+2 C_{m}(j)\right]+C_{m}(i)\right\}
$$

The estimated cost of a location update operation in hierarchical scheme is given as Eq. (6). In hierarchical architecture, when a mobile moves into new location area from the old location area, the tracking information should be updated at all servers on the path from new to least common ancestor of old and new, which explains the first term in Eq. (6), Especially when the mobile moves farther away from its home server, the associated servers to extend the tracking path should be updated, which explains the second term in Eq. (6).

$$
\begin{aligned}
M_{\text {hierarchical }}= & P\left(m_{a, n}=1\right) C_{m}(1)+P\left(m_{a, n}=2\right)\left\{C_{m}(2)+2 C_{m}(1)\right. \\
& +4 T(1,2)\}+\sum_{i=3}^{R} P\left(m_{a, n}=i\right)\left\{2 C_{m}(2)+2 C_{m}(1)\right. \\
& \left.+4 T(1,2)+\sum_{j=2}^{i-1} 4 T(j, j+1)\right\}
\end{aligned}
$$

The estimated cost of the location search procedure in the Multi HLR scheme is given by:

$$
\begin{aligned}
R_{\text {multiHLR }}= & P\left(m_{s, d}=1\right) \times C_{m}(1)+\sum_{i=2}^{L} P\left(m_{s, d}=i\right) \times \\
& \left(C_{m}(1)+C_{m}(L)+4 T(1, L)+\right. \\
& \delta \times\left\{\left\{C_{m}(1)+C_{m}(L)+4 T(1, L)\right\}+\right. \\
& \theta \times\left[\sum_{i=L+1}^{R} P\left(m_{s, d}=i\right) \times\left(\sum_{j=L}^{i-1} 4 T(j, j+1)+C_{m}(L)\right)\right] \\
& \left.+(1-\theta) \times\left[\sum_{i=L+1}^{R} P\left(m_{s, d}=i\right)\right] \times\left(\sum_{j=L}^{i-1} 8 T(j, j+1)+2 C_{m}(L)\right)\right\} \\
& \text { where } \\
\delta= & 1-\sum_{i=1}^{L} P\left(m_{s, d}=i\right)
\end{aligned}
$$

The first part of Eq.7 describes the cost of the location search procedure when the call is originated from and terminated at the covering area of the same HLR. The second part illustrates the cost of this procedure when the call is originated from and terminated at the covering areas of two different HLRs.

According to database driven scheme, the call is relayed to callee's residing layer 1 server through the shortest path on the tree between caller and callee. The estimated cost of a location search in the database driven scheme is given by:

$$
R_{\text {Databassedriven }}=\sum_{i=1}^{R} P\left(m_{s, d}=i\right) \times\left[\sum_{j=1}^{i-1}\left\{4 T(j, j+1)+2 C_{m}(j)\right\}+C_{m}(i)\right]
$$

The estimated cost of the location search procedure of the HLR/VLRs scheme is given by:

$$
R_{H L R / V L R}=\sum_{i=1}^{R} P\left(m_{s, d}=i\right) \times\left\{C_{m}(1)+C_{m}(R)+4 T(1, R)\right\}
$$

The estimated cost of the location search procedure of the hierarchical scheme is given by:

$$
\begin{aligned}
& R_{\text {heirarchical }}=P\left(m_{s, d}=1\right)\left[P\left(m_{s, d}=1\right) C_{m}(1)+P\left(m_{s, d}=2\right)\left\{2 C_{m}(1)\right.\right. \\
& \left.\left.+C_{m}(2)+4 T(1,2)\right\}+\sum_{i=3}^{R} P\left(m_{s, d}=i\right)\left\{2 C_{m}(1)+2 C_{m}(2)+4 T(1,2)+T(1, i)\right\}\right] \\
& +\sum_{i=2}^{R} \sum_{j=1}^{2} P\left(m_{s, d}=i\right) P\left(m_{s, d}=j\right)\left\{C_{m}(1)+C_{m}(2)+4 T(1,2)+T(1, i)\right\} \\
& +\sum_{i=2}^{R} \sum_{j=3}^{R} P\left(m_{s, d}=i\right) P\left(m_{s, d}=j\right) \times\left\{C_{m}(1)+2 C_{m}(2)+4 T(1,2)+T(1, i)+T(1, j)\right\}
\end{aligned}
$$

The first term in the above equation is the delivery cost at all servers on the path from the least common ancestor server of mobile home server and callee's current residing location to callee's location area. The second and third terms are related to the cost consumed at the remaining servers on the delivery path from caller's current residing location to callee's current residing location for mobile terminated call.

\section{RESULTS}

The numerical values for all the four schemes i.e multi HLR, conventional HLR/VLR, database driven and hierarchical schemes are evaluated and compared. Different values of $\mathrm{R}$ and $\mathrm{L}$ (as per figure 6) are considered in order to study the impact of the location of the new HLRs on the performance of the multi HLR model.

\section{Assumptions:}

In this analysis it is assumed that the database access cost in layer $i$ is equal to $i$, the cost of crossing a link between layer $i-1$ and layer $i$ is equal to $i$ and value of $\beta$ and $\theta$ have an equal probability values.

$$
\text { For comparison purpose } \mathrm{M}_{\text {multiHLR }} /
$$

$\mathrm{M}_{\mathrm{HLR} / \mathrm{VLR}}, \mathrm{M}_{\text {database driven }} / \mathrm{M}_{\mathrm{HLR} / \mathrm{VLR}}, \mathrm{M}_{\text {hierarchical }} / \mathrm{M}_{\mathrm{HLR} / \mathrm{VLR}}$ are denoted as the relative cost of location update procedure for the multi HLR model, the database driven and hierarchical model to that of the HLR/VLR model, respectively. These costs are obtained using equation no. 3 to 6 and shown in figure 11 and 12. A similar analysis is also conducted on location search procedure. $\mathrm{R}_{\text {multi HLR }} / \mathrm{R}_{\mathrm{HLR} / \mathrm{VLR}}, \mathrm{R}_{\text {database driven }} /$ $R_{\text {HLR/VLR }} R_{\text {hierarchical }} / R_{\text {HLR/VLR }}$ be the relative cost of location search procedure for the multi HLR model, the database driven ad hierarchical model to that of the HLR/VLR model, respectively. These costs are obtained by using equation no. 7 to 10 and shown in fig 13 and 14. A relative cost of 1 means that the cost under both the models are the same.

Figure 11 shows the performance of the analyzed scheme with $R=5$ and various values of $L$ (i.e. $L=$ $2,3,4)$. In the figure users are classified with respect to their moves. When $\mathrm{p}$ (probability value that MU move is intra 
VLR) is very small (i.e. the mobile unit moves are not local), the HLR/VLR scheme outperforms the multi HLR scheme, the database driven and hierarchical schemes because a greater number of signaling message is exchanged between the HLRs and VLRs in the multi HLR scheme as well as between the various databases in the database driven and hierarchical schemes. The database driven scheme is most costly. When $\mathrm{p}$ increases, a significant cost reduction is obtained with the multi HLR scheme when $\mathrm{L}=2$ and 3 . Less saving is obtained when $\mathrm{L}=4$ because the location of new HLRs become closer to the root (where the HLR is located in HLR/VLR scheme). When $p=1$, the performance of all the four schemes are equal. This is normal situation since when $\mathrm{p}=1$, the Mobile unit's move are always local. It can be observed that the performance of database driven and hierarchical schemes improves when $p$ increases significantly ( $p>0.6)$. It is just because of decrease in the number of signaling messages during the location update when the mobile unit moves become local.

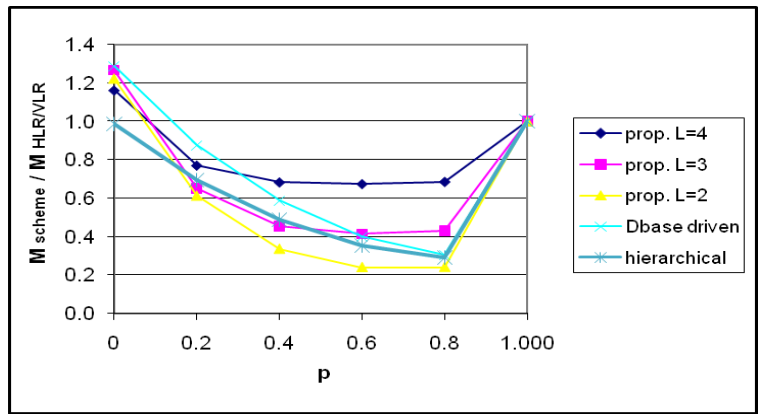

Figure 11 : Relative cost for location update when $R=5$

The figure 12 shows the performance of all the four schemes when $R=3$. When $R=3$ and $L=3$ the multi HLR scheme resembles the HLR/VLRs but when $\mathrm{L}=$ 2 the multi HLR scheme always outperforms the database driven and hierarchical schemes, however, hierarchical seems to be better than database driven.

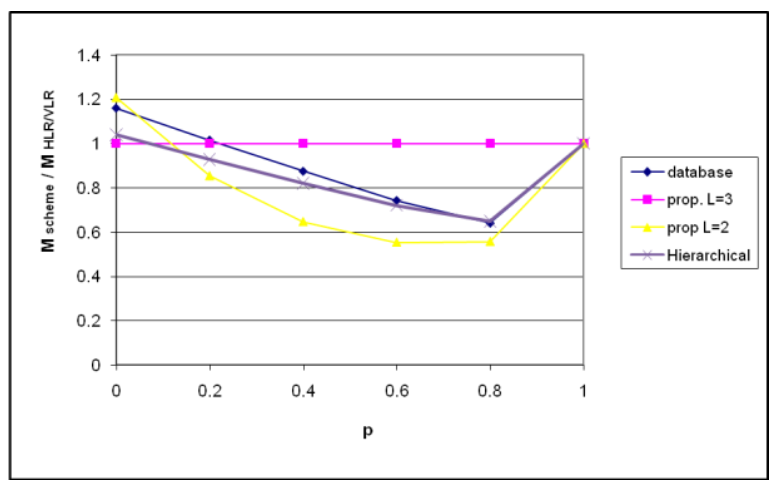

Figure 12 : Relative cost for location update when $\mathrm{R}=3$

A similar analysis is conducted on the location search procedure. Users are classified by their location when processing an incoming call. Figure 13 shows the performance of location search procedure in all the four schemes when $\mathrm{R}=5$. In this case it is observed that in almost all the circumstances, the multi HLR scheme results in a significant cost reduction when $\mathrm{L}=2$ and $\mathrm{L}=3$ and always outperforms the database driven and hierarchical schemes. The cost reduction is at its peak when $\mathrm{q}$ approaches 1 , since in this case the probability, that the called LA and the calling LA are served by the same HLR, increases. The figure 13 and figure 14 shows that a maximum cost reduction is obtained with the multi HLR scheme when $\mathrm{L}=2$. The reduction decreases when the value of $L$ increases since the new HLRs become closer to the HLR of the HLR/VLR architecture.

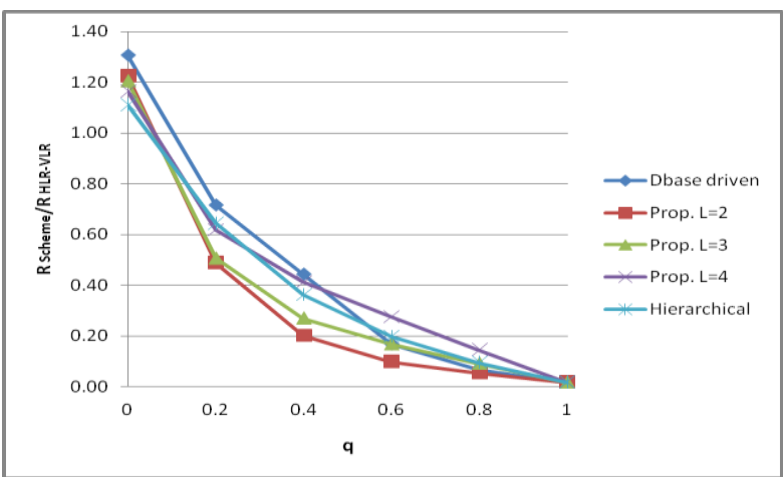

Figure 13 : Relative cost for location search when $R=5$

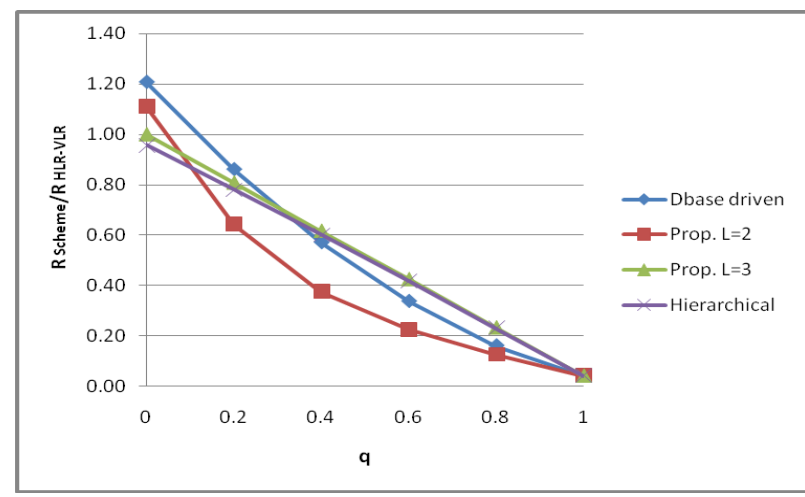

Figure 14 : Relative cost for location search when $\mathrm{R}=3$

So, based on the analysis done above, It can be concluded that the performance of location management scheme is highly dependent on users' mobility and incoming call characteristics. Further, the class of users for which the multi HLR scheme yields a net reduction in signaling traffic and databases loads have also been investigated. Users are classified by their Call-to-Mobility ratio (CMR), which is defined as the ratio between the average number of calls to an Mobile unit per unit time and the average number of times this Mobile unit changes LAs per unit time (i.e, incoming call rate/mobility rate).

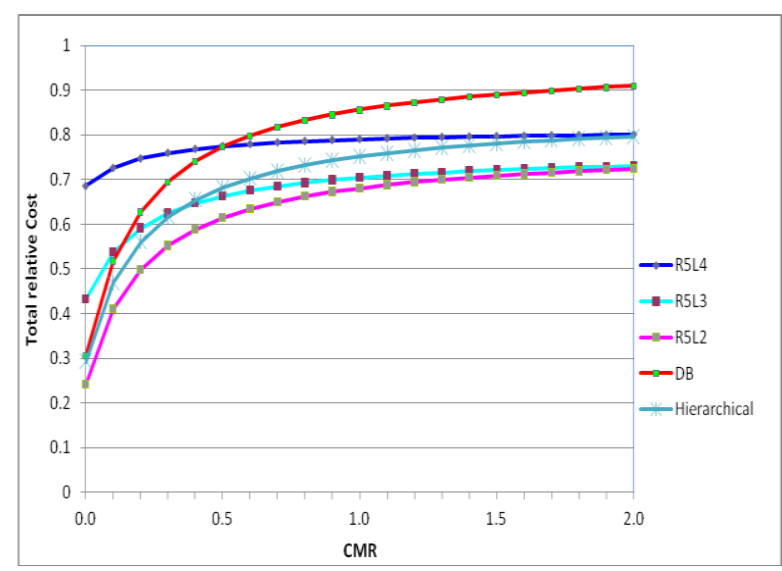

Figure $15(\mathrm{a})$ : Total Relative cost when $\mathrm{p}=0.8$ and $\mathrm{q}=0.1$ 


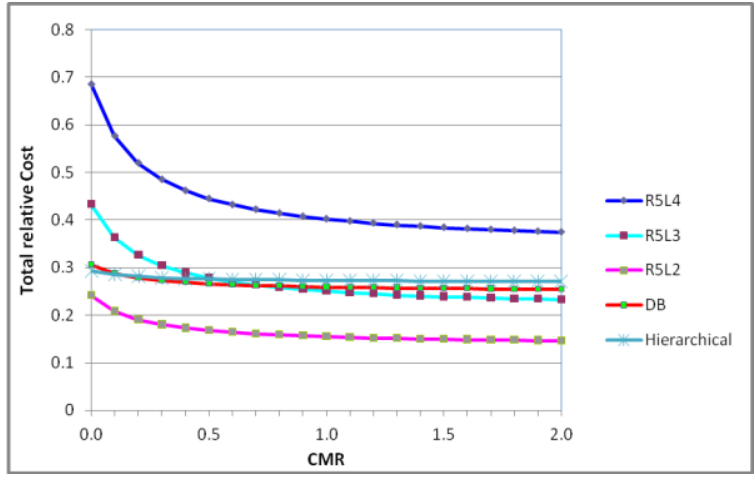

Figure 15(b): Total Relative cost when $\mathrm{p}=0.8$ and $\mathrm{q}=0.5$

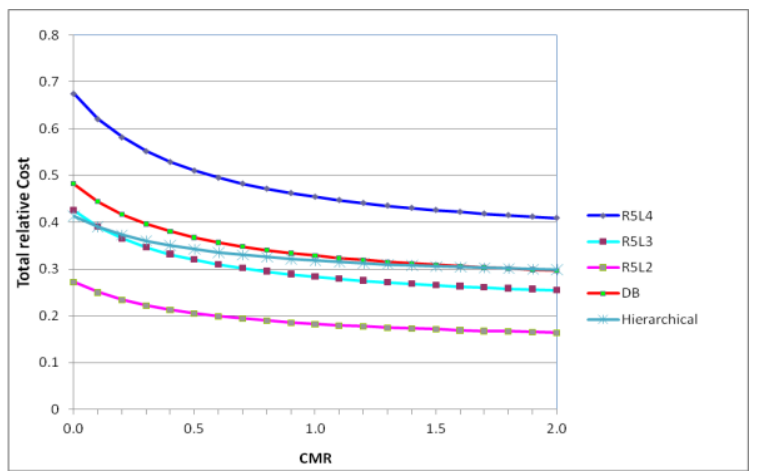

Figure 15(c) : Total Relative cost when $\mathrm{p}=0.5$ and $\mathrm{q}=0.5$

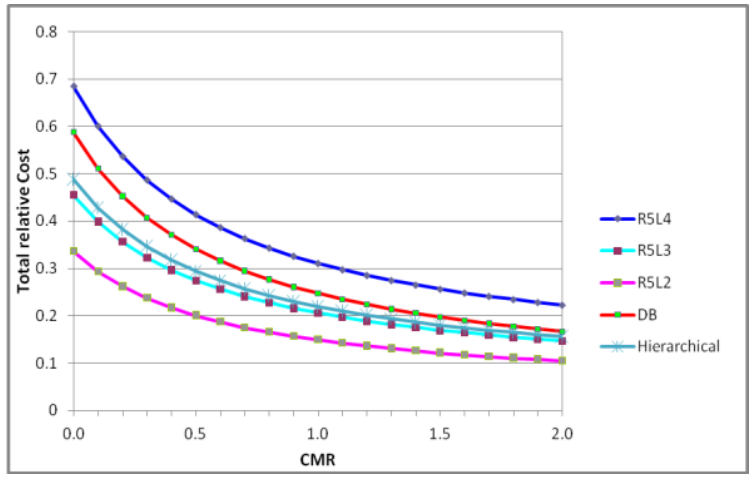

Figure 15(d) : Total Relative cost when $\mathrm{p}=0.4$ and $\mathrm{q}=0.9$

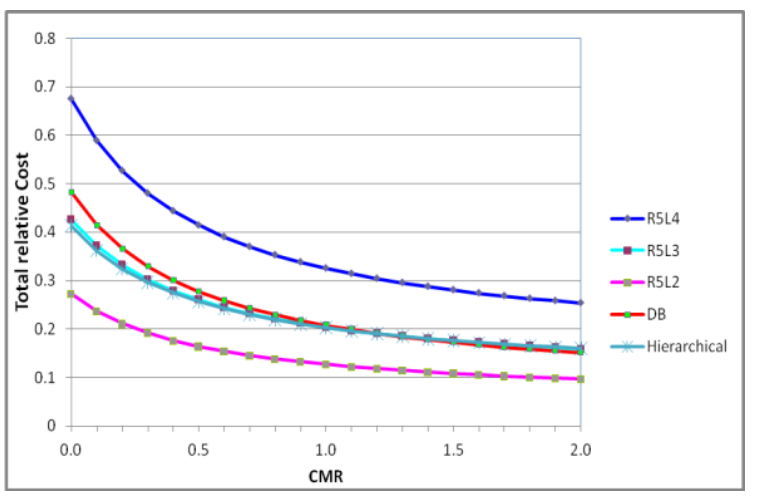

Figure 15(e): Total Relative cost when $p=0.5$ and $q=0.8$

Then it follows that :

$$
\mathrm{CMR}=\lambda / \mu
$$

Where, $\lambda$ is the incoming call rate and $\mu$ is the mobility rate. The total estimated cost per unit time for location update and location search in HLR/VLR, database driven, hierarchical and multi HLR scheme are denoted as CHLR/VLR, Cdatabase driven, Chierarchical, and Cmulti HLR respectively. It follows that :

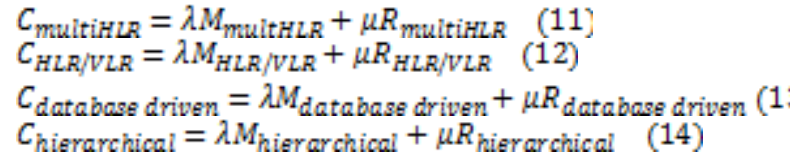

Finally, the total relative cost of the multi HLR location management scheme can be defined as the ratio of the total cost per unit time for the multi HLR scheme to that of HLR/VLRs architecture. Similarly, the relative cost of the database driven and hierarchical schemes to that of HLR/VLRs architecture. These costs can be derived from Eqs 11-14 as follows:

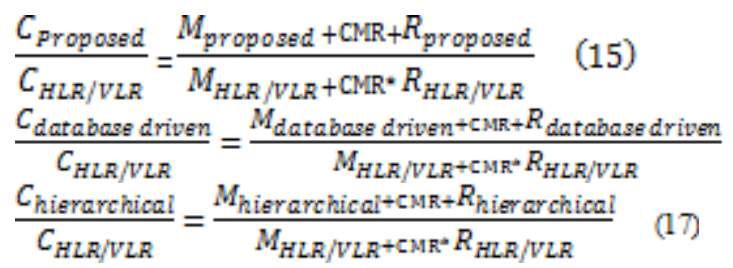

Figure 15 shows the total relative of the multi HLR scheme, database driven and hierarchical schemes plotted against CMR using various values of $p, q, L$. The CMR value varies from 0.0 to 2.0. In Figure 15 (a) when $\mathrm{p}=0.8$ (i.e., moves are often local) and $\mathrm{q}=0.1$ (incoming calls are most probably not local), the multi HLR scheme yields a remarkable reduction at $\mathrm{L}=2$ in comparison to $\mathrm{L}=$ $3 \& 4$ and other schemes. From the graph of Figure 15 (a) the multi HLR scheme at $\mathrm{L}=2$ seems to be the best compared to other schemes and hierarchical have better performance than the database driven scheme. The database driven scheme seems to be worst in most of times, however, it is better than multi HLR only when the CMR $=0.2$, but as the value of CMR increases the value shoots to give worst result.

Figure 15 (b) shows the total relative of the multi HLR scheme, database driven and hierarchical schemes plotted against CMR for $\mathrm{p}=0.8$ and $\mathrm{q}=0.5$. In Figure 15(a) the value of $q$ is 0.1 so, in this case when $q$ increases $(q=0.5$ i.e., incoming calls are mixture of local and non-local), the multi HLR scheme continues to outperform the HLR/VLRs scheme in all the cases and the database driven \& hierarchical scheme in most of the cases. The hierarchical scheme seems to be better than database scheme when CMR is less than 0.2 but as value of CMR increases the database outperforms the hierarchical scheme. Multi HLR scheme at $\mathrm{L}=3$ performs better only when the value of CMR is more than 1 otherwise it shows poor performance than others. The other discussed scheme shows better performance to multi HLR scheme only when $\mathrm{L}=4$ (since, new HLRs become closer to the root).

Figure 15 (c) shows total relative cost when $\mathrm{p}=0.5$ and $\mathrm{q}=0.5$ (mobile unit's moves and incoming call are mixture of local and non-local), the multi HLR scheme outperforms the HLR/VLR scheme. I also outperforms database driven and hierarchical scheme when $\mathrm{L}=2$ and $\mathrm{L}=3$. Hierarchical scheme shows its performance in between database scheme and multi HLR scheme at $\mathrm{L}=3$ as it is showing similar characteristic with multi HLR $(L=3)$ at CMR below 0.2 and with database at CMR above 1.5 . Multi HLR shows worst performance at $\mathrm{L}=4$ as new HLRs approaches closer to the root. 
Figure 15(d) shows that when $\mathrm{p}=0.4$ and $\mathrm{q}=0.9$ (i.e mobile unit moves are often not local and incoming calls are often local), the multi HLR scheme outperforms other schemes when $\mathrm{L}=2$ and $\mathrm{L}=3$. However, when $\mathrm{L}=4$, the performance of the multi HLR scheme is poorer than that of the database driven scheme and hierarchical scheme in all the cases. However performance of hierarchical scheme is better than database driven. When $\mathrm{p}$ increases to 0.5, Figure 15(e), the multi HLR scheme results in a significant cost reduction compared to other schemes at $\mathrm{L}=2$ but performance of hierarchical is similar to multi HLR at $\mathrm{L}=3$ and better than database driven when $\mathrm{CMR}<1$. At $\mathrm{L}=4$ multi HLR again shows poorer performance however better than HLR/VLR scheme.

\section{CONCLUSION AND FUTURE WORK}

By the analysis done in the last section, it is observed that the multi HLR scheme eliminates most of the drawbacks of HLR/VLR, database driven and hierarchical schemes. So it can be concluded that multi HLR scheme perform better than all schemes for location management discussed so far.

Further, we have made an investigation of the classes of users for which the multi HLR scheme results in net reduction in signaling traffic and databases loads. This is based on classification of users by their call-tomobility ratio (CMR), From the graph drawn between total relative cost vs. CMR (Figure 15) it is clear that for the value of $\mathrm{L}=2$ and 3 the multi HLR scheme outperform the database driven and hierarchical schemes.

Performance analysis done in this work shows that the multi HLR architecture is potentially beneficial for large class of users and can result in substantial reductions in total user location management cost. The work done by us can further be extended in future to address other issues like handoff management, wireless security and the impact of mobility on transaction management.

\section{REFERENCES}

[1] EINTIA, "Cellular radio-telecommunications intersystem operations," Technical Report IS-41 Revision B, EIA/TIA, 1991.

[2] S. Mohan and R. Jain, "Two user location strategies for personal communications services," IEEE Personal Commun., pp. 42-50, First Quarter 1994.

[3] M. Mouly and M. B. Pautet, "The GSM system for mobile communications," Palaiseau, France, 1992.

[4] R. Jain, Y. B. Lin, and S. Mohan, "A caching strategy to reduce network impacts of PCS," IEEE J. Select. Areas Commun., vol. 12, no. 8, Oct. 1994.

[5] J. Ho and F. Akiyildiz, "Local Anchor Scheme for reducing Signaling Cost in Personal Communication Networks," ," IEEE/ACM Transaction on Networking., vol. 4, no. 5, pp. 709-725 Oct. 1996.

[6] R. Jain and Y. B. Lin, "An auxiliary user location strategy employing forwarding pointers to reduce network impact of PCS," ACM-Baker J.
Wireless Network, vol. 1, no. 2, pp. 197-210, July 1995.

[7] W. Ma, Y. Fang, "Two Level Pointer Forwarding Strategy for Location Management in PCS Networks," IEEE/ACM Trans. On Mobile Computing, vol. 1, no. 1, January - March 2002.

[8] S. Rangarajan, K. Ratnam, and A. T. Dahbura, "A fault-tolerant protocol for location directory maintenance in mobile networks," in Proc. of the 25th International Symposium on Fault-Tolerant Computing, June 1995.

[9] K. Ratnam, I. Matta, and S. Rangarajan, "A fully distributed location management scheme for large PCS," Tech. Rep. BU-CS-1999-010, Computer Science Department, Boston University, Boston, MA 02215, August 1999.

[10] Bong-sue Suh, Jin-seek Choi, Jae-kyoon Kim, "Design and performance analysis of hierarchical location management strategies for wireless mobile communication systems." Computer Communications, 23 (2000) 550-560.

[11] K. Ratnam, I. Matta, and S. Rangarajan, "Analysis of caching-based location management in personal communication networks," in IEEE ICNP '99, 1999.

[12] Haidar Safa, Samuel Pierre, "A New Architecture for Improving Location Management in PCS Networks," Journal of Computer Sciencel (2): 249-258, 2005.

[13] Anantharam, V., M.L. Honig, U. Madhow and V.K. Wei, 1994. Optimization of a database hierarchy for mobility tracking in a personal communications network. Performance Evaluation, 20 287-300.

[14] Mao, Z. and C. Douligeris, 2004. A distributed database architecture for global roaming in nextgeneration mobile networks. IEEE/ACM Transactions on Networking, 12: 146-160.

[15] Wang, J.Z., 1993. A fully distributed location registration strategy for universal personal communication systems. IEEE J. Selected Areas Commun., 11: 850-860.

[16] Vijay Kumar, Mobile Databases, JOHN WILEY \& SONS, INC., PUBLICATIO 